\title{
Delay tolerant firework routing: a geographic routing protocol for wireless delay tolerant networks
}

\author{
Anna Sidera ${ }^{1 *}$ and Stavros Toumpis ${ }^{2}$
}

\begin{abstract}
We present the Delay Tolerant Firework Routing (DTFR) protocol, a protocol designed for use in disconnected Delay Tolerant Networks (DTNs) that consist of a very large number of location-aware, highly mobile nodes. Networks with these properties appear frequently in many settings, notably in vehicular networks. Under DTFR, each data packet travels from the source to the estimated location of the destination using high-priority transmissions and a delay-tolerant variant of geographic forwarding. Once there, a number of packet replicas are created, and the replicas proceed to travel through the area where the destination is expected to be. Using simulations in an urban setting, we compare DTFR with two baseline protocols (Flooding and Spray and Wait), one recently proposed state-of-the-art protocol (GeoDTN+Nav), and an idealistic protocol of our design which we term Bethlehem Routing (BR). For a wide range of environmental parameters, DTFR performs significantly better than the other realistic protocols, in terms of throughput and delay, and close to the upper performance bounds of BR. We also develop an analytical framework based on stochastic geometry tools, a number of simplifying assumptions, and a small number of judiciously chosen approximations. Using this framework, we develop approximate closed form expressions for the average end-to-end throughput and delivery delay of DTFR and BR.
\end{abstract}

\section{Introduction}

In Delay Tolerant Networks (DTNs), the delay in the delivery of the data is much larger than typically expected, and in fact it is comparable to the time it takes the topology to change. A number of DTN applications have recently been proposed in various wireless settings $[1,2]$ and the Internet [3]. Furthermore, a number of theoretical studies have shown that a tradeoff exists between the packet delay and the throughput $[4,5]$. A significant amount of work has also been devoted to the design of practical routing protocols [6-8].

In this study, we present Delay Tolerant Firework Routing (DTFR), a protocol for performing routing in DTNs. DTFR is designed for use in networks where the number of nodes is very large (in the orders of thousands and tens of thousands) and where nodes move with large speeds. It requires that nodes are capable of knowing their

\footnotetext{
*Correspondence: sidera.anna@ucy.ac.cy

1 Department of Electrical and Computer Engineering, University of Cyprus, 75 Kallipoleos Avenue, 1678 Nicosia, Cyprus

Full list of author information is available at the end of the article
}

location, and the location of their destinations (possibly with an error) through the combined use of GPS receivers (or a similar technology) and a location service. DTFR is superior to other protocols we compare it with when the network is disconnected but not very sparse. A notable domain where all these assumptions frequently hold is vehicular ad hoc networks (VANETs) [9-11].

Broadly speaking, DTFR works as follows: When a packet is created, the source uses information about the location of its destination, provided from a location service and/or previously received data packets, to create an estimate about the destination's current location, which we call the Firework Center (FC). The source sends a single copy of the packet to the $\mathrm{FC}$, using high-priority transmissions and a novel delay-tolerant geographic forwarding rule, which we term Greedy Lazy Forwarding (GLF). Under GLF, if the current holder of a packet sees another node closer to the destination, it forwards the packet to that node, otherwise the current holder takes no action, and just waits for one such node to appear. Once

\section{Springer}

(c) 2013 Sidera and Toumpis; licensee Springer. This is an Open Access article distributed under the terms of the Creative Commons Attribution License (http://creativecommons.org/licenses/by/2.0), which permits unrestricted use, distribution, and reproduction in any medium, provided the original work is properly cited. 
the packet arrives at the FC, a number of replicas are created, which proceed to propagate in various directions, again using GLF, systematically covering the area where the destination might be.

In Figure 1, we plot an example trajectory of a packet while it is traveling to the $\mathrm{FC}$, and the trajectories of the replicas created after the FC is reached. In the figure, we have denoted a transmission from a transmitter to a receiver with a line segment connecting their two locations. Observe that the line segments do not form a continuous trajectory; rather, the trajectory appears disconnected. Such disconnections are due to the fact that, under GLF, nodes do not have to transmit immediately a packet they receive. Therefore, such disconnections represent extended sojourns of the packet at a node while the packet waits for a suitable relay to appear. Observe that, collectively, these trajectories trace a pattern similar to the pattern created by a 'palm tree' firework [12], hence the name of our protocol.

The remainder of this study is organized as follows: in Section 2, we discuss DTN and VANET protocols related to this study. In Section 3, we discuss our basic assumptions on the network. In Section 4, we present the DTFR protocol in detail. Section 5 focuses on the performance evaluation of DTFR by simulation, and its comparison with two baseline protocols [Spray and Wait (SW) [7]

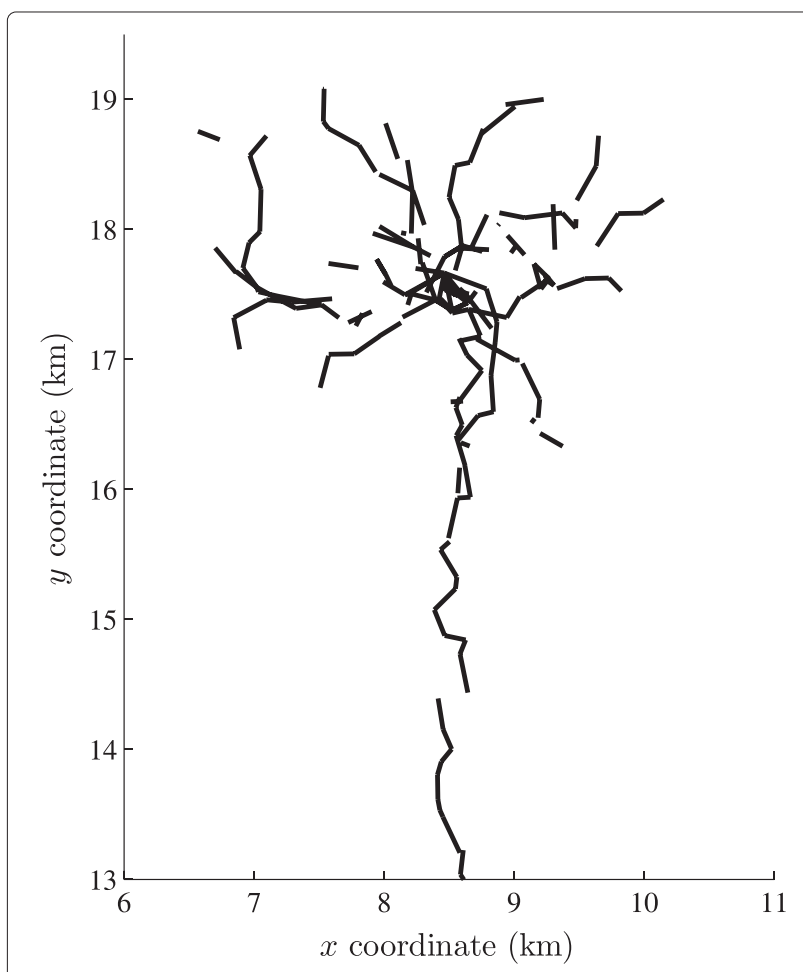

Figure 1 Example trajectory of a packet and its replicas, being routed with DTFR. The source is outside the figure. and Flooding], a state-of-the-art protocol (GeoDTN+Nav [11]), and Bethlehem Routing (BR), an idealized protocol of our own design. In Section 6, we provide approximate closed form expressions for the average throughput and delay under DTFR and BR. We conclude in Section 7. The more technical parts of the analysis are placed in the Appendix.

\section{Related work}

In [7], the Spray and Wait (SW) protocol is proposed. SW consists of two phases. In the Spray Phase, the source distributes $L$ copies to $L$ distinct relays. In the Wait Phase, the relays move around the network, until eventually one of them meets the destination and hands over its replica of the packet. Our protocol also employs replicas, however, here, the replicas are created not at the location of the source, but at a location estimated to be close to the destination, in order to conserve bandwidth. In addition, nodes make use of geographic information.

The Mobility-centric approach for Data Dissemination in Vehicular networks (MDDV) protocol [13] is based on two phases. During the Forwarding Phase, the message travels to the destination region, and then, in the Propagating Phase, it is distributed to all nodes there. In the Forwarding Phase, a group of nodes are forwarding the message along a trajectory consisting of road segments chosen by the protocol. The group consists of the nodes that estimate that they are near the message head which is the node closest to the destination region along the trajectory. The members of the group change as the message propagates or the vehicles move. Nodes estimate the position of the message head based on information that is inserted in the copies of the packet, by nodes that estimate they might be the message head. In DTFR, the packet also travels to the location of the destination, during a Homing Phase, but using GLF and high-priority transmissions. In addition, during the Homing Phase, there is only a single copy of the packet at any time, and if the node that has that copy moves away from the FC, it still has to forward the copy. Finally, our use of replicas is more efficient than the Propagating Phase of MDDV, which distributes the packet to all nodes in the destination region.

Greedy Perimeter Stateless Routing (GPSR) [14] uses a combination of greedy forwarding on the full network graph and perimeter forwarding on a planarized network graph, i.e., a subgraph of the original graph with no crossing links. Initially, the packet is forwarded on the full network graph using the greedy mode; if, at some point, there is no neighbor closer to the destination than the node holding the packet, the packet enters the perimeter mode, traversing the faces of the planarized network graph using the right-hand rule [14]. If the packet, while in perimeter mode, reaches a node closer to the destination 
than the point at which the packet entered the perimeter mode, the packet switches back to the greedy mode.

Lochert et al. [9] propose Greedy Perimeter Coordinator Routing (GPCR), a protocol designed for use in vehicular networks. GPCR is based on the observation that the road network creates a naturally planar graph that can be exploited for communication purposes. Both greedy routing and perimeter routing are executed using that graph. However, GPCR suffers from the problem that when there is no node at a junction, packets will be forwarded across that junction, and this might lead to a routing loop.

To alleviate this problem, the GeoCross protocol is introduced in [10]. GeoCross is similar in its operation to GPCR, but its perimeter mode is enhanced and capable of detecting and removing crossing links and creating a planar graph.

GeoDTN+Nav [11] consists of the greedy and perimeter modes of GeoCross and a third mode, termed the DTN mode, which can deliver packets even in the absence of end-to-end routes. In GeoDTN+Nav, packets are first forwarded using the greedy mode and, when this fails, using the perimeter mode. If the perimeter mode also fails, the protocol finally switches to the DTN mode and relies on mobility to deliver packets. To decide when to switch to the DTN mode, a node uses a cost function related to network partition detection and to the navigation information of its neighbors. When a packet is in the DTN mode, it returns to the greedy mode whenever it encounters a node that is closer to the expected location of the destination than the point where the perimeter mode started.

DTFR and GeoDTN+Nav are related, as they both employ a geographic routing mode and a DTN mode. However, they have a number of key differences. First, GeoDTN+Nav makes use of a perimeter mode, which DTFR avoids, in order to conserve bandwidth, and in order to avoid the routing loops associated with running a perimeter mode in a network of highly mobile nodes. Second, GeoDTN + Nav was designed without taking into account links between nodes that are not on the same road and so makes no use of potentially useful links between nodes lying on different roads. Third, in GeoDTN+Nav, the packet only travels to the expected destination position inserted in the packet by the source, whereas, in DTFR, if the destination is not found when the packet reaches its expected position, replicas are employed to find it. Also, the rules for entering the greedy mode from other modes are different. Finally, DTFR uses a set of priority rules for gaining access to the medium. As we show in the simulation section, all these differences lead to significant deviations in the performance of the two protocols.

More recently, in [15], Location-Aided ROuting for DTNs (LAROD) has been proposed. Like DTFR, LAROD makes use of a delay tolerant geographic routing protocol. In particular, each node carrying a packet (termed a custodian) periodically broadcasts it to its neighborhood. Nodes closer to the destination that overhear the transmission set up a timer that depends on their location. After its timer expires, a node broadcasts a reply informing its own neighborhood that it is the new custodian. If the original custodian, or a node that received the packet and waits for its timer to expire, listens to a reply from a node in its neighborhood announcing that it is the new custodian, it discards the packet. This algorithm is related but different from our GLF algorithm, notably allowing the packet to be propagated along multiple paths. Another fundamental difference between LAROD and DTFR is that DTFR creates multiple replicas once the FC is reached. This makes DTFR more robust to destination localization errors.

We note that the term 'Firework' has also been used in Peer-to-Peer Networking [16,17] where a content-based 'Firework Query Model' is proposed. Also, in [18] a multicast protocol called 'Fireworks Routing' is presented, for use in a general, non-DTN, multicast wireless ad hoc setting. This protocol organizes multicast group members into cohorts. One group member in each cohort is selected to be a cohort leader. Cohort leaders establish a sparse multicast tree among themselves and the source and they use broadcasting to deliver the packets to other group members in their cohort. Although Fireworks Routing and DTFR have a number of similarities, they also have key differences. DTFR applies a delay tolerant version of geographic routing, avoids broadcasting in order to conserve bandwidth, and prioritizes transmissions. Also, in DTFR no multicast structure is maintained and the packet is not given to nodes near a cohort leader but to nodes in a geographic region where the destination is estimated to be. As the works in [16-18] appear in very different contexts, a meaningful comparison with them is not possible.

We note that a preliminary version of this study appeared, in conference version, in [19]. With respect to [19], this study introduces the analytical framework of Section 6 and a larger set of simulation results.

\section{Basic network assumptions}

In this section, we outline our fundamental assumptions on the network. Although these assumptions are satisfied in a variety of settings, a good example are large vehicular DTNs $[11,20]$.

\subsection{Nodes}

We assume a very large number of nodes, on the orders of thousands and tens of thousands. We assume that the nodes move in a region independently of each other, and independently of their communication needs, with potentially very high speed. These assumptions imply that any 
protocol used must be scalable and robust, and preclude, for example, proactive routing, as well as the use of mechanisms that are sensitive to frequent topology changes, such as perimeter routing [14].

\subsection{Communication needs}

Nodes are executing one or more applications that depend on the communication between node pairs. (One member of the pair could be an Access Point, or similar entity, communicating with multiple nodes.) The application(s) running at each node are delay tolerant; however, there is a maximum acceptable delay for the delivery of the packets.

\subsection{Localization}

Each node is capable of knowing its own location, either directly (e.g., through GPS) or indirectly (e.g., using beacons). We assume that there is a mechanism available to the nodes that provides the location of their destination, possibly with error. The design of such a mechanism is of great practical interest, but, as the issues of routing and localization are not tightly related, we choose to focus only on the issue of routing. However, we do take into account the fact that localization information may have errors, and any routing algorithm should be robust with respect to these.

\subsection{Neighborhood awareness}

Each node is aware of the network topology in its local neighborhood. At the very least, this means that the node is aware of all nodes close enough for direct communication with them to be possible. In the more general case, each node might also have location information about some of the nodes a small number of hops away. Neighborhood awareness is achieved through the use of eavesdropping packets and/or beaconing [21].

\subsection{Connectivity level}

In [22], wireless ad hoc networks are classified in four categories: (i) end-to-end paths exist almost always, (ii) end-to-end paths exist for some fraction of the time, (iii) the network is always partitioned, (iv) multi-hop paths are rare. DTFR is designed for use in categories (ii) and (iii) of the above classification.

To clarify this point, Figure 2 shows simulation results for networks of 10,000 and 100,000 nodes. The nodes are placed randomly, according to the uniform distribution, on a square area. Nodes that are separated by a distance of at most $R$ can communicate directly with each other. The nodes are divided randomly and uniformly in pairs and each node is the destination for the packets of its counterpart. In the figure, we plot the average Reachability, which is defined as the proportion of nodes that have a path to their destination, versus the expected node degree. (We trace different values of the expected node degree by

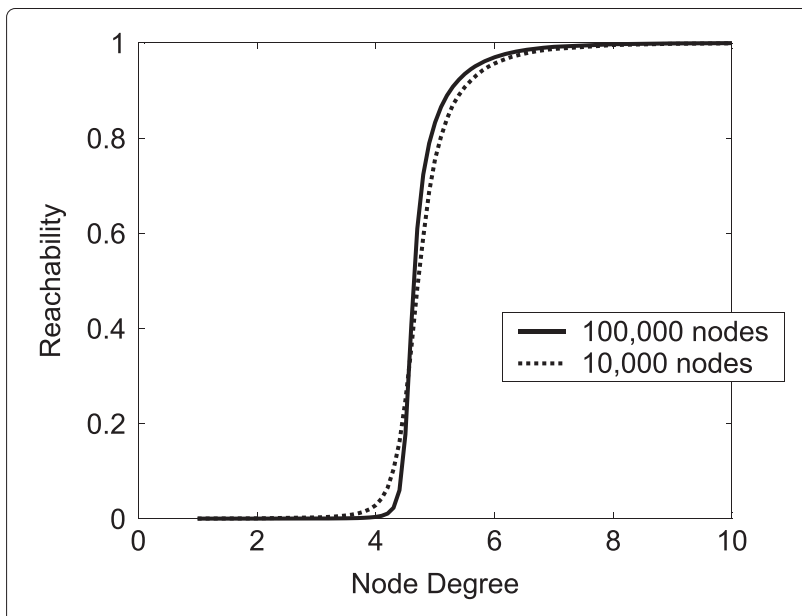

Figure $\mathbf{2}$ Reachability versus node degree.

varying the communication range $R$.) As the figure shows, for values of the average node degree below a threshold, an end-to-end path to the destination does not always exist. Therefore, traditional ad hoc routing protocols such as greedy-face-greedy protocols [14] are not suitable and DTN solutions are needed. We will show that for a large range of average node degrees at this case, for some values of the maximum acceptable delay for the delivery of the packets, DTFR performs better than SW and the other protocols we simulate.

\section{The DTFR protocol}

The DTFR protocol consists of four mechanisms: (1) a Dissemination Rule, responsible for disseminating a number of replicas in the vicinity of the destination, (2) a forwarding rule, responsible for node-to-node packet forwarding, which we term Greedy Lasy Forwarding (GLF), (3) a Priorities Policy, for assigning priorities to nodes contending for access to the wireless medium, and (4) a Buffer Policy. We now discuss each of these. We stress that some implementation details of these mechanisms will depend on the details of the application, and so are left undefined here.

\subsection{Dissemination rule}

The dissemination rule of DTFR consists of four phases:

1. Homing Phase: The packet travels to a point called the Firework Center (FC), at the center of a region where the source estimates the destination to be.

2. Explosion Phase: Then, the packet is replicated and given to $L$ relays.

3. Spread Phase: Then, the packet replicas travel, using GLF, to $L$ different points called the Firework Endpoints (FEs), that are symmetrically placed around the FC, at a distance $D$ from it. Once there, the replicas are discarded. The distance $D$ is chosen 
to be such that the destination will be between the FC and the FEs with high probability.

4. Lock Phase: At any time during the first three phases, if a packet comes near enough to the destination to discover a multihop route, it enters the Lock Phase wherein it is forwarded to the destination using that route, in the usual, non-DTN manner.

\subsection{Greedy Lazy Forwarding (GLF)}

GLF is used in the Homing and Spread phases, when packets travel toward the FC and FEs, respectively. Consider a node $A$ holding a packet $P$, destined for some distant location $D$. Let the forwarding area $F$ be the set of points closer to the destination $D$ than point $A$, and also with a distance of at most $R$ from $A$. (Note that when $D$ is far away from $A$, $F$ becomes a semicircle.) Node $A$ uses the following rule for choosing the next relay of $P$ :

1. (Greedy part) If there is at least one node within $F$, then $A$ forwards the packet to one of the nodes in $F$. (Details of the choice are left to the particular implementation.)

2. (Lazy part) If there is no node within $F$, then $A$ waits until a node appears on the boundary of $F$, and then immediately sends the packet to that node.

This rule is being executed continuously: when a packet arrives at a node, the node checks the appropriate forwarding area for potential relays. If the area contains one or more nodes, the packet gets forwarded to one them. Otherwise, the packet waits for one such node to appear. Observe that, as with all other geographic routing protocols, our forwarding protocol is greedy, in the sense that it provides an immediate improvement if this is possible. However, in contrast to them, it is also lazy: upon failure to achieve an immediate improvement, it just waits for the topology to get better.

\subsection{Firework center and firework edges calculation}

The locations of the FC and the FE are specified by the packet source when the packet is created, and inserted to the packet's header.

As with other aspects of DTFR, the precise method for calculating the FC depends on the details of the environment and the application, and notably the localization mechanism that is assumed to exist (see Section 3). The overriding principle is that the FC should be as close as possible to the destination, when the packet arrives at the FC. Therefore, if the localization mechanism provides to the packet source an estimate of the location of the destination at a recent time instant, then this estimate should be used as an FC. If, however, the localization mechanism also provides estimates of the location of the destination at future instances (e.g., by revealing the route of the destination and its basic travel characteristics), then the FC can be selected so that it approximately intercepts the destination some time in the future [23]. If the source and destination participate in a regular exchange of packets, then they can provide to each other all the information about their location and future trajectory that is available to them.

The $L$ FEs are placed uniformly on the circumference of a circle of radius $D$ centered at the FC. $D$ is selected to be, with a high degree of confidence, large enough so that one of the replicas will have the opportunity to overtake the destination. Clearly, the larger $D$ is selected, the larger $L$ should also be, in order for the circular region bounded by the FEs to be covered adequately. Note that using excessively large values for $L$ and $D$ means that bandwidth will be wasted. The precise rule for selecting the values of $L$ and $D$ will depend on the environment, and notably on the mobility model, and so is omitted here.

\subsection{Priorities policy}

In order to access the medium, packets are given different priorities, depending on the phase they are in. Lock Phase transmissions have priority over transmissions of all other phases. This is because when a packet goes near its destination we do not want to loose the opportunity to deliver it, given the changing topology. Homing Phase transmissions have priority over Spread Phase transmissions and Explosion Phase transmissions, as we do not want to delay the only copy of a packet from reaching the FC and so delay the search in the whole region near its destination. Explosion Phase transmissions have priority over Spread Phase transmissions, as we want to create all replicas quickly.

\subsection{Buffer policy}

The buffer of each node has a finite size $B$. Once a buffer is full, the node cannot receive any packet unless it is destined for that node, and must discard the packets its user creates. Packets are discarded when they reach the FEs. Also, the packets have a time-to-live (TTL) equal to the maximum acceptable delay for the delivery of the packets.

\subsection{Bethlehem routing}

Under Bethlehem Routing (BR), each packet is continuously aware of the location of its destination, and moves towards it by continuously staying in the Homing Phase with the actual location of the destination chosen as the FC. Once near enough to the destination to discover a route, the packet will enter the Lock Phase. Excluding this (crucial) modification, BR is identical to DTFR. Clearly, BR can only work when a fast location service is available to the system. In relatively small networks, such a location service has been shown to exist: Kuiper and Nadjm-Tehrani [15] found no significant difference 
between an oracle location service and the practical location service LoDiS introduced there, for networks with approximately 100 nodes. This indicates that BR might be a practical and better routing protocol than DTFR, when the number of nodes is on that order. Note, however, that networks with 10,000 nodes are within the scope of this study, and the performance of BR we report here, for networks of this size, should be viewed as an upper bound only.

\section{Simulations}

\subsection{Simulations tool}

In order to evaluate our protocol, we have developed Very Large DTN Simulator (VL-DTN-S), a simulation tool specifically designed for DTNs, and written in C. The tool is available online [24].

We have refrained from using NS-3 [25], OMNeT++ [26], or a similar general purpose simulation tool, because such tools were designed for routing in traditional networks and so are not best adapted to the unique challenges appearing in DTNs (e.g., the need for very large buffers), particularly in the case where there are many thousands of nodes. We also refrained from using ONE [27], DTNSim2, or any other JAVA-based DTN simulation tool, as the use of JAVA necessarily slows down the execution of the simulation when the number of nodes is very large. Discussions on the relative merits of the various simulators for use in DTN environments can be found in $[27,28]$, and references therein.

Efforts have been made to make VL-DTN-S as accurate as possible. Among others, (i) full buffer information for all nodes is kept, (ii) realistic physical layers are used, and (iii) contention in the channel is taken into account. At the same time, efforts have been made so that the simulator is as fast as possible and, as a result, the tool is capable of detailed simulations of networks of more than $10^{4}$ nodes on a desktop computer, and for a variety of routing protocols. Challenging simulations with $10^{4}$ nodes take at most a few hours.

\subsection{Simulation setting \\ 5.2.1 Mobility model}

Nodes move on a square grid composed of vertical and horizontal roads. Initially, each node is placed at random on the grid, and then proceeds to travel, using the road network, to a randomly chosen location, using a constant speed, uniformly distributed between 0 and $v_{\max }$. Then, it chooses another random location, and another speed, moves to that location, and so on.

\subsubsection{Traffic pattern}

All nodes are divided in pairs, each node communicating with its counterpart. Pairs do not change for the whole duration of the simulation.

\subsubsection{Channel model}

We assume an urban environment where both Line Of Sight (LOS) and Non-LOS (NLOS) communication are possible, however the power of signals received through a LOS attenuates slower with distance. In particular, LOS communication is only possible between nodes lying on the same road. However, if a node is within a threshold distance $R_{T}$ from the intersection between two roads, we assume that this node belongs to both roads. $\left(R_{T}\right.$ is essentially the radius of the junction.)

In the case of LOS transmissions, the signal power $P_{r}$ received at distance $d$ from a transmitter is

$$
P_{r}=P_{0}\left(\frac{d_{0}}{d}\right)^{\alpha_{\mathrm{LOS}}}
$$

and, for NLOS transmissions,

$$
P_{r}=P_{0}\left(\frac{d_{0}}{d}\right)^{\alpha_{\mathrm{NLOS}}}
$$

where $P_{0}$ is the received power at a small reference distance $d_{0}$ from the transmitter and $\alpha_{\mathrm{LOS}}$ and $\alpha_{\text {NLOS }}$ are exponents that describe the environment, typically 2-6 [29], with $\alpha_{\mathrm{LOS}}<\alpha_{\mathrm{NLOS}}$, so that NLOS signals attenuate faster.

\subsubsection{Transmitter model}

While transmitting, a node cannot listen to the transmissions of other nodes. If node $k$ is not a transmitter, a packet from node $i$ is received successfully at node $k$ if

$$
\frac{P_{i k}}{\sum_{j \in S, j \neq i} P_{j k}+N}>\gamma_{T},
$$

where $N$ is the background noise, $\gamma_{T}$ is the minimum Signal-to-Interference plus Noise Ratio (SINR) required at the receiver, $P_{j k}$ is the received power at node $k$ from node $j$, and $S$ is the set of all transmitters.

\subsubsection{Slotted time}

We slot time, and at the start of each timeslot each node creates a packet with a predefined probability $\lambda$. The packet is immediately stored in the buffer if it is not full. The transmission of each packet takes one timeslot. Timeslots are assumed to be so short, that the topology cannot change appreciably for a timeslot duration, and hence channel gains are constant throughout each of them. As the networks we simulate have many thousands of node, for such a slotted system to work it is necessary to employ GPS receivers or, alternatively, a sophisticated distributed clock synchronization system [30]. Further discussion on this topic goes beyond the scope of this study.

\subsubsection{Medium Access Control}

At the start of each slot, nodes employ a MAC scheme to decide who will transmit at that slot, what packet, and 
to whom. At any given time during the execution of this scheme, the state of a node can be either available or reserved. At the start of the slot, all nodes are available, but progressively attempt to make reservations, according to their priorities (see Section 4.4). For a node $A$ to be able to send a packet to another node $B$, both $A$ and $B$ must not be reserved. If this is the case, nodes $A, B$ and all the nodes within distance $K \cdot d_{A B}$ from $A$ or $B$ become reserved. $K$ is a constant greater than 1 , which we term the Reservation Radius Constant. As we are not interested in the evaluation of the MAC layer, we assume that the reservations are all arranged instantaneously, at the start of each slot, and no MAC control messages are simulated. As our focus is on routing, we refrain from using a more detailed MAC protocol. We note, however, that our MAC protocol allows the use of priorities and realistically captures the capabilities of the wireless channel, notably modeling congestion.

\subsubsection{Power control}

If node $A$ has decided to transmit a packet to another node $B, A$ uses a power level $P_{t}$ such that the transmission will be successful if the interference from competing transmissions turns out to be at most $\left(I_{f}-1\right)$ times the thermal noise, where $I_{f}$ is a constant we call the Power Control Safety Margin. Also, there is a maximum allowed transmission power $P_{0 \max }$.

\subsubsection{Local routing table}

As already discussed, nodes maintain a routing table that can be used for routing in their immediate neighborhood. To conserve bandwidth and improve robustness, nodes do not use local routes that minimize the number of hops. Rather, a link cost is introduced, and nodes try to use paths with minimum total link cost. In particular, each LOS transmission from a node $A$ to another node $B$ is associated with a cost $d_{A B}^{2}$, where $d_{A B}$ is the distance between nodes $A$ and $B$. Each NLOS transmission is associated with a cost $d_{A B}^{\frac{2 \alpha_{\mathrm{NLOS}}}{\alpha_{\mathrm{LOS}}}}$. The routing table includes destinations for which there is a path with total link cost at most equal to a threshold value $C_{T}$, which we term the Local Routing Threshold.

We do not simulate control messages for the creation of the local routing table. Therefore, interference experienced by data packets comes only from data packets. We believe that, as we are interested in the more challenging case of communication across large distances, these assumptions, that essentially remove local routing issues from the picture, are justified.

\subsubsection{Firework Center}

In the case of DTFR, GeoDTN+Nav, and GeoCross, we assume that when a packet is created the source is informed of the location of its destination and uses that as the FC. Unless stated otherwise, we assume that this location is reported with no error.

\subsubsection{Routing protocols}

We simulate DTFR, BR, GeoDTN+Nav, GeoCross, SW, and Flooding. We also simulate a protocol that we call Bethlehem GeoDTN+Nav (BetGeo), which is identical to GeoDTN+Nav except from one point: whenever a routing decision is made that involves the location of the destination, instead of using the position that the destination occupied at the time of the packet's creation, its current position is used. As with the Bethlehem protocol, this is an idealization, however the performance of this protocol allows us to evaluate the cost on the performance of GeoDTN+Nav of using location information that is not current. Under the flooding protocol, each node sends copies of all packets it has in its buffer to all nodes it meets, and all transmissions have the same priority.

In our implementation of SW, and in order to have a more fair comparison to DTFR, nodes make use of the local routing table. Lock Phase transmissions have priority over Spray Phase transmissions.

In our implementation of GeoCross and GeoDTN+Nav, nodes are given access to the local routing table. Lock Phase transmissions have priority over all other transmissions. Greedy mode transmissions, perimeter mode transmissions, and DTN mode transmissions are equal in priority, but transmissions from junction nodes have priority over transmissions from street nodes.

Unless otherwise stated in each particular case, the parameters used are those of Table 1. For each point in the plots, we simulate each protocol for different values of its various parameters, and select the values that produce the best results. The results shown are for the steady state of the simulation. We also run the mobility model until it reaches its steady state, before starting creating packets.

\subsection{Results}

In Figure 3a, we show the packet delivery ratio versus the packet arrival rate for all protocols. Even with very small arrival rates, no protocol manages to deliver all packets within the TTL. This is due to the fact that the network is often partitioned for periods of time comparable to or larger than the TTL. In addition, quite often the network is not partitioned but bottlenecks are formed due to the topology, leading to queueing delays.

Observe that the delivery rate of GeoDTN+Nav is significantly smaller than the delivery rate of DTFR. There is a number of reasons for this. First of all, DTFR uses the Explosion, Spread, and Lock Phases to counter the fact that the destination is moving. No similar mechanisms exist in GeoDTN+Nav. (Note, however, that even with Bethlehem GeoDTN+Nav, where GeoDTN+Nav is enhanced so that the packets have continuous perfect 
Table 1 Default simulation parameters

\begin{tabular}{ll}
\hline Parameter & Numerical value \\
\hline Slot duration & $0.01 \mathrm{~s}$ \\
Packet arrival rate & $\lambda=0.02$ packets $/ \mathrm{s} /$ node \\
Number of nodes & $n=5000$ \\
Side of the grid in which the nodes move & $7 \mathrm{~km}$ \\
Distance between junctions & $200 \mathrm{~m}$ \\
Junction radius & $R_{T}=10 \mathrm{~m}$ \\
Maximum node speed & $V_{\max }=10 \mathrm{~m} / \mathrm{s}$ \\
Packet TTL & $6 \mathrm{~min}$ \\
LOS exponent & $\alpha_{\mathrm{LOS}}=3$ \\
Non-LOS exponent & $\alpha_{\mathrm{NLOS}}=5$ \\
Propagation model reference distance & $d_{0}=1 \mathrm{~m}$ \\
SINR threshold & $\gamma_{T}=10$ \\
Power control safety margin & $I_{f}=10$ \\
Thermal noise over transmitter power & $\frac{N}{P_{0 \mathrm{max}}}=1.25 \cdot 10^{-9}$ \\
Local routing threshold & $C_{T}=4 \cdot 10^{4} \mathrm{~m}^{2}$ \\
Simulation duration & $1 \mathrm{~h}$ \\
Buffer size & $B=10^{4}$ packets \\
Reservation radius constant & $K=2.5$ \\
Maximum location error & $E=0 \mathrm{~m}$ \\
\hline
\end{tabular}

knowledge of the position of their destination, the delivery ratio improves modestly over GeoDTN+Nav.) Second, under GeoDTN+Nav it is possible that packets leave the perimeter mode and enter the DTN mode at a node that is further away from the destination than the node they were when they entered the perimeter mode. In between, they were transmitted multiple times, wasting precious bandwidth in the process. DTFR, on the other hand, never transmits a packet away from the destination. Third, under GeoDTN+Nav packets stay in the DTN mode even when there are neighbors of the current holder closer to the destination, because their distance to the destination is greater than the distance between the destination and the point where the packet entered the perimeter mode. Under DTFR, on the other hand, nodes always send packets to neighbors closer to the destination than themselves.

In Figure 3b, we show the packet delivery ratio versus the network size. We change the network size by changing the number of nodes and the dimensions of the area, keeping the number of nodes per unit road length constant. Note that the performance of all protocols diminishes with the network size. This is due to the facts that the TTL counter remains fixed, that with larger network sizes partitions are more frequent, and that with larger network sizes more bandwidth is consumed for the transmission of each packet.
In Figure 3c, we show the packet delivery ratio versus the transmission range. We change the transmission range by changing the value of $\frac{N}{P_{0 \max }}$. All protocols gain by an increase in the transmission range; however, the two protocols that do not depend on the fast forwarding of the packets to the area where the destination is expected, SW and Flooding, benefit the least. On the other hand, SW is slightly superior to the rest (except Flooding) in the case of small transmission ranges. The performance of GeoCross and GeoDTN+Nav increases fast as the transmission range increases, because the perimeter mode becomes more efficient for larger transmission ranges.

In Figure 3d, we show the packet delivery ratio versus the maximum speed of the nodes. For high speeds, SW gives good performance. Clearly, when the node mobility is too high, the best strategy for the source is to get out as many replicas as possible. Also observe that, in the other extreme, when nodes are immobile, GeoCross gives better results than DTFR. This is expected: when a packet reaches a local optimum, and nodes are immobile, waiting is futile, and the only alternative is going into perimeter mode. However, for all the cases in the middle, DTFR is surpassed only by BR. Note that to obtain the points in Figure $3 \mathrm{~d}$ for 0 velocity, we averaged multiple runs of the simulation, each of them for a different network topology chosen randomly from steady state topologies.

In our simulations, we place the FC at the location of the destination at the time of the creation of the packet. By the time the packet arrives at the FC, the destination has moved away. In order to keep packet losses at small levels, it is important that the distance $D$ between the FC and the FEs is large enough. In Figure 4, we plot the empirical cumulative distribution function of the distance $x$ between the FC and the destination at the time of the arrival of a packet at the FC. The plot was created using a simulation with the parameters of Table 1 and $10^{4}$ packets. Packets that entered the Lock Phase or expired before arriving at the $\mathrm{FC}$ were disregarded. The average distance is a little over $200 \mathrm{~m}$, and the distance is less than $1000 \mathrm{~m}$ for around 95\% of the packets. Most importantly, the distribution of the distance had a very thin tail, which means that $D$ does not have to be set excessively large to accommodate a wide range of distances $x$. In this simulation, the number of FEs was 16, and they were placed $2000 \mathrm{~m}$ from the FC.

Until now, we have assumed that the source obtains the exact location $\left(x_{0}, y_{0}\right)$ of the destination at the time the packet is created using a location service, and inserts that location in the packet. Assume now that the $X$ and $Y$ coordinates inserted in the packet by the source are uniformly distributed in the intervals $\left[x_{0}-E, x_{0}+E\right]$ and $\left[y_{0}-E, y_{0}+E\right]$, respectively, where $E$ is called the Maximum Location Error. The performance metrics as a 


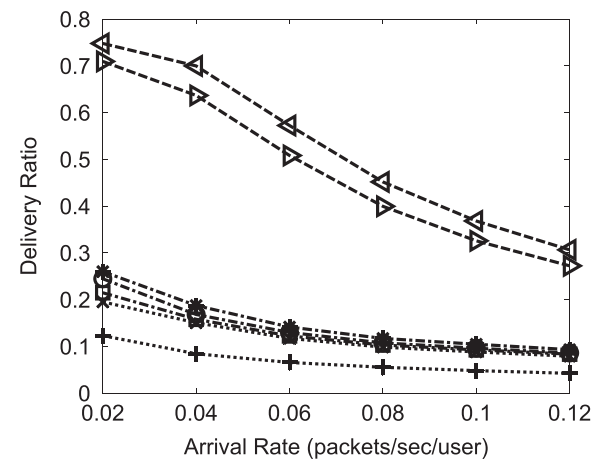

(a)

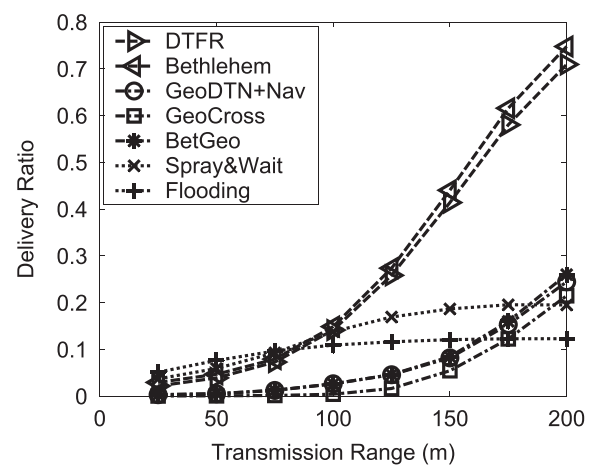

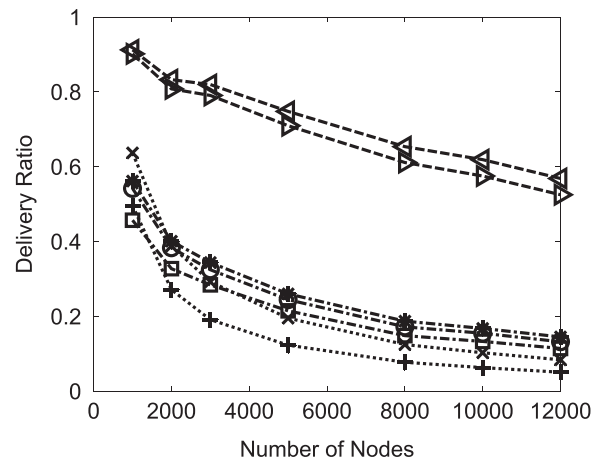

(b)

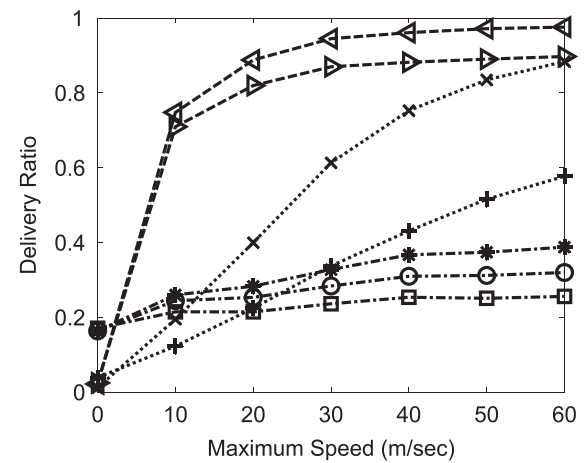

Figure 3 Comparison of DTFR with other protocols. The legend of Plot (c) applies to all plots. Therefore, for example, the + marker denotes the Flooding protocol in all six plots.

function of $E$ are shown in Figure 5. Note that the performance of all protocols using the location of the destination decreases as $E$ increases, but the performance of DTFR is superior to that of the others even for large values of $E$.

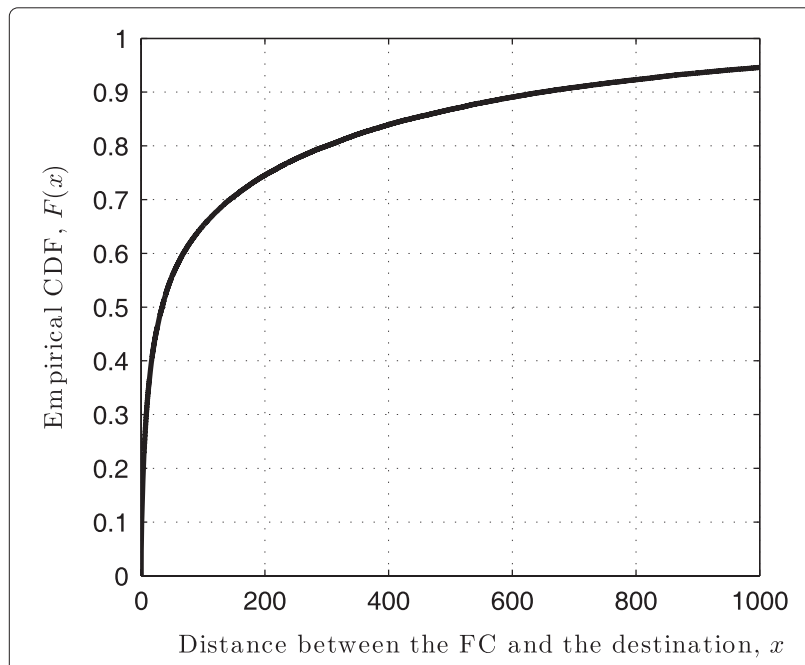

Figure 4 Empirical Cumulative Distribution Function of the distance between the $\mathrm{FC}$ and the destination at the time of the arrival of the packet at the FC.
We also simulated GeoCross using the parameters of Table 1 but with a very low arrival rate of $10^{-4}$ packets/s/node, immobile nodes, and a very large number of permitted hops in the perimeter mode, $h_{\max }=10^{4}$. It was found that only $36 \%$ of the packets reached their destination, although an end-to-end path existed for $45 \%$ of the node pairs. Therefore, although GeoCross is a major breakthrough over GPCR, it does not altogether eradicate routing loops.

\section{Analysis}

In this section, we present a succinct analysis of DTFR and $\mathrm{BR}$, and in particular calculate the average delay and per node throughput achieved by DTFR and BR. Due to the complexity of these protocols, it is necessary to make a number of simplifying assumptions and approximations. Therefore, the aim of this section is not to arrive at accurate values for the performance metrics, as was done using simulations in Section 5. Rather, our analysis has the following goals: (i) to verify the fundamental effects of the basic parameters of the environment (such as the node density and node speed) on the performance of the protocols, that were observed in the simulations, and (ii) to shed light on the fundamental reasons for its superior performance to protocols such as SW for a wide range of node 

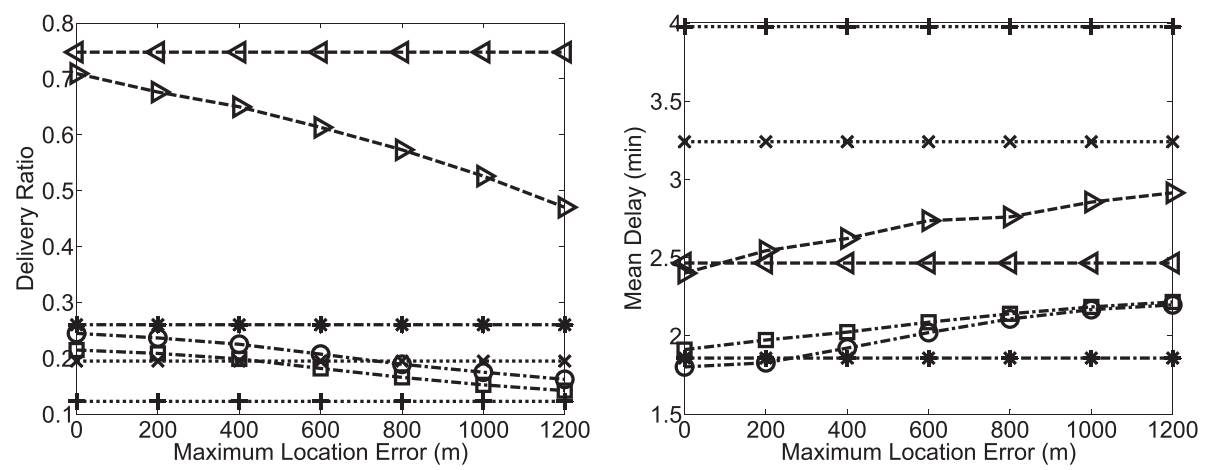

Figure 5 Performance versus maximum location error. The legend of Figure $3 c$ applies. Therefore, for example, the + marker denotes the Flooding Protocol.

degrees. The notation used in the analysis is summarized in Table 2.

\subsection{Network model}

\subsubsection{Node placement and mobility}

The nodes are placed on an infinite region according to a spatial Poisson process with density $\lambda$ (therefore, there is an infinity of nodes). All nodes move with a velocity of magnitude $v_{0}$, each one on its own direction, which is kept constant. Movement directions are independent and uniformly distributed in $[-\pi, \pi]$.

\subsubsection{Data traffic}

Each node sends data to another node chosen randomly among the rest, so that the distance between a source and its destination is a random variable with first moments $E\left(D_{o d}\right)$ and $E\left(D_{o d}^{2}\right)$.

\subsubsection{Channel access}

All nodes are equipped with a transceiver of data rate $r_{D}$ bps, and the maximum distance of direct communication is $R$. As we are interested in modeling very large

\section{Table 2 Notation of Section 6}

\begin{tabular}{|c|c|c|c|}
\hline Node density & $\lambda$ & Node speed & $v_{0}$ \\
\hline Transceiver data rate & $r_{D}$ & Distance between OD pair & $D_{o d}$ \\
\hline Delay of hop $i$ & $D_{i}$ & $\begin{array}{l}\text { Maximum communication } \\
\text { range }\end{array}$ & $R$ \\
\hline Cost of hop $i$ & $C_{i}$ & Progress of hop $i$ & $X_{i}$ \\
\hline Event that $F$ is empty & $M$ & Forwarding area & $F$ \\
\hline $\begin{array}{l}\text { Normalized transmis- } \\
\text { sion cost }\end{array}$ & $c_{p}$ & Packet speed & $v_{p}$ \\
\hline $\begin{array}{l}\text { Average cost of proto- } \\
\operatorname{col} x\end{array}$ & $E\left(C^{x}\right)$ & $\begin{array}{l}\text { Average delay of protocol } \\
x\end{array}$ & $E\left(D^{x}\right)$ \\
\hline $\begin{array}{l}\text { Radius of explosion } \\
\text { phase }\end{array}$ & $R_{X}$ & $\begin{array}{l}\text { Maximum per-node } \\
\text { throughput of protocol } x\end{array}$ & $T^{x}$ \\
\hline
\end{tabular}

delays, comparable to the time needed for the topology to change substantially, we assume that the packet transmission time is 0 .

To capture the contention among the nodes for the shared channel we assume that, for a transmission from a transmitter $A$ to a receiver $B$ to be successful, there must be no transmitter or receiver $C$ closer to receiver $B$ than transmitter $A$. Therefore, we associate with each successful transmission across distance $d_{i}$ a disk-shaped footprint of radius $d_{i}$ centered at the receiver. The footprints are not allowed to overlap, in order for the transmissions not to interfere, hence the condition above. This model for channel access contention is simple, and ignores many aspects of wireless communication, notably the fact that interference is additive. However, it captures the fact that there is a tradeoff between the number of transmissions and the distances they cover $[4,5,31]$. A similar model was used in [31]. We define the cost of a transmission across distance $d$ to be $\pi d^{2}$.

\subsubsection{Other assumptions}

Under BR, each packet is constantly aware of the destination location. Under DTFR, each packet becomes aware of the destination location at the moment of its creation, but receives no update after that point. Nodes are equipped with buffers of infinite size. Also, nodes do not maintain any local routing protocol, and only know the location of the nodes currently within their communication range $R$.

\subsection{Delay, progress, and cost of first hop}

Let node $A$ create, at time $t=0$, a packet destined for location $Z$. We assume that $A$ is at the origin, and the destination $Z$ on the positive $x$-axis, and sufficiently far away so that the forwarding region $F$ is a semicircle. We also assume that if there are more than one nodes in $F$ the next hop is chosen at random. (This choice of next hop was used in [32].)

Let $D_{1}$ be the delay until the packet is forwarded to its first relay, $B$, and let $\left(X_{A}, Y_{A}\right)$ and $\left(X_{1}, Y_{1}\right)$ be the 
coordinates of $A$ and $B$ at time $D_{1}$. Finally, let $X_{T}=$ $X_{1}-X_{A}, Y_{T}=Y_{1}-Y_{A}$, and $C_{1}=\pi\left(X_{T}^{2}+Y_{T}^{2}\right)$ be the transmission cost. Observe that $X_{1}$, which we will call progress, represents the net reduction of the distance to the destination achieved at the conclusion of the first hop.

Let the event $M$ that when $A$ creates the packet, $F$ is empty. Conditioning on $M$,

$$
\begin{aligned}
& E\left(D_{1}\right)=E\left(D_{1} \mid M\right) P(M)+E\left(D_{1} \mid M^{\prime}\right)(1-P(M)), \\
& E\left(X_{1}\right)=E\left(X_{1} \mid M\right) P(M)+E\left(X_{1} \mid M^{\prime}\right)(1-P(M)), \\
& E\left(C_{1}\right)=E\left(C_{1} \mid M\right) P(M)+E\left(C_{1} \mid M^{\prime}\right)(1-P(M)) .
\end{aligned}
$$

As $F$ has an area $\pi R^{2} / 2$, it follows that $P(M)=$ $\exp \left[-\lambda \pi R^{2} / 2\right]$.

Observe that if $F$ is empty, the first relay $B$ is the first node to enter it. In the Appendix, we show that

$E\left(D_{1} \mid M\right)=\frac{I_{1}}{v_{0} R \lambda}, \quad E\left(X_{1} \mid M\right)=I_{2} R, \quad E\left(C_{1} \mid M\right)=I_{3} R^{2}$,

where $I_{1} \simeq 0.4817, I_{2} \simeq 0.3890, I_{3} \simeq 2.3317$.

Now assume that $M^{\prime}$ holds. We first note that, as transmission do not take time, we have $E\left(D_{1} \mid M^{\prime}\right)=0$. As the first relay $B$ is chosen randomly among those available, it follows that its location is uniformly distributed in $F$. Therefore

$$
\begin{aligned}
E\left(X_{1} \mid M^{\prime}\right) & =\frac{1}{\pi R^{2} / 2} \iint_{F} x d A \\
& =\frac{2}{\pi R^{2}} \int_{0}^{R}\left(\int_{-\pi / 2}^{\pi / 2} r^{2} \cos \theta d \theta\right) d r=\frac{4}{3 \pi} R, \\
E\left(C_{1} \mid M^{\prime}\right) & =\frac{1}{\pi R^{2} / 2} \iint_{F} \pi r^{2} d A \\
& =\frac{2}{R^{2}} \int_{0}^{R}\left(\int_{-\pi / 2}^{\pi / 2} r^{3} d \theta\right) d r=\frac{\pi R^{2}}{2} .
\end{aligned}
$$

Therefore, we know all quantities appearing on the right-hand sides of Equations (2), (3), and (4).

\subsection{Packet speed and normalized cost in greedy/lazy routing}

Let $P$ be a packet traveling from node $A$ to node $B$, along a sequence of hops $i=1, \ldots$ Let $D_{i}, X_{i}$, and $C_{i}$ be the delay, progress, and cost of hop $i$. We make the following assumption $^{\mathrm{a}}$ :

Basic Assumption: The $\left\{D_{i}\right\}$ are i.i.d., the $\left\{X_{i}\right\}$ are i.i.d., and the $\left\{C_{i}\right\}$ are i.i.d.
The law of large numbers applies and we have, as $n \rightarrow$ $\infty$,

$$
\begin{aligned}
& \frac{1}{n} \sum_{i=1}^{n} D_{i} \rightarrow E\left(D_{1}\right), \quad \frac{1}{n} \sum_{i=1}^{n} X_{i} \rightarrow E\left(X_{1}\right), \\
& \frac{1}{n} \sum_{i=1}^{n} C_{i} \rightarrow E\left(C_{1}\right) .
\end{aligned}
$$

Taking quotients,

$$
\frac{\sum_{i=1}^{n} X_{i}}{\sum_{i=1}^{n} D_{i}} \rightarrow \frac{E\left(X_{1}\right)}{E\left(D_{1}\right)}, \quad \frac{\sum_{i=1}^{n} C_{i}}{\sum_{i=1}^{n} X_{i}} \rightarrow \frac{E\left(C_{1}\right)}{E\left(X_{1}\right)} .
$$

The first limit expresses the fact that the speed of the packet, averaged over the whole duration of its journey, will converge to the packet speed

$$
v_{p} \triangleq \frac{E\left(X_{1}\right)}{E\left(D_{1}\right)}=v_{0} \lambda R^{2}\left[\frac{I_{2}}{I_{1}}+\frac{4\left(1-\exp \left(-\lambda \pi R^{2} / 2\right)\right)}{3 \pi I_{1} \exp \left(-\lambda \pi R^{2} / 2\right)}\right] .
$$

The packet speed equals the rate with which the packet approaches the destination, and its value is not affected by any move that the packet does perpendicularly to the direction to the destination. In other words, it is the magnitude of the projection of the average velocity vector of the packet on the line connecting the current location of the packet to the destination. It is taken to be positive when the packet moves toward the destination.

The second limit expresses the fact that the total cost over the total progress converges to the normalized cost

$$
c_{p} \triangleq \frac{E\left(C_{1}\right)}{E\left(X_{1}\right)}=R \times \frac{I_{3} \exp \left(-\lambda \pi R^{2} / 2\right)+\frac{\pi}{2}\left(1-\exp \left(-\lambda \pi R^{2} / 2\right)\right)}{I_{2} \exp \left(-\lambda \pi R^{2} / 2\right)+\frac{4}{3 \pi}\left(1-\exp \left(-\lambda \pi R^{2} / 2\right)\right)} .
$$

We make the approximation that the speed with which packets move is $v_{p}$, and the cost per unit of distance is $c_{p}$, even when the number of hops $n$ does not approach infinity.

Observe that $v_{p}$ is proportional to $v_{0}$, and depends on the node density $\lambda$ and communication range $R$ only through the average number of neighbors, $\lambda \pi R^{2}$. In Figure 6 , we plot $v_{p} / v_{0}$ as a function of $\lambda \pi R^{2}$. The speed $v_{p}$ is an increasing function of $\lambda \pi R^{2}$ and becomes larger than $\nu_{0}$ for $\lambda \pi R^{2} \simeq 1.64$. The plot reveals that the packet speed is significantly larger than the node speed for a wide range of node degrees for which the network is not connected (compare Figure 6 with Figure 2).

\subsection{Delay and throughput of DTFR}

We have defined $D_{o d}$ as the random distance between an origin and a destination of a packet. Let $R_{X}$ be the 


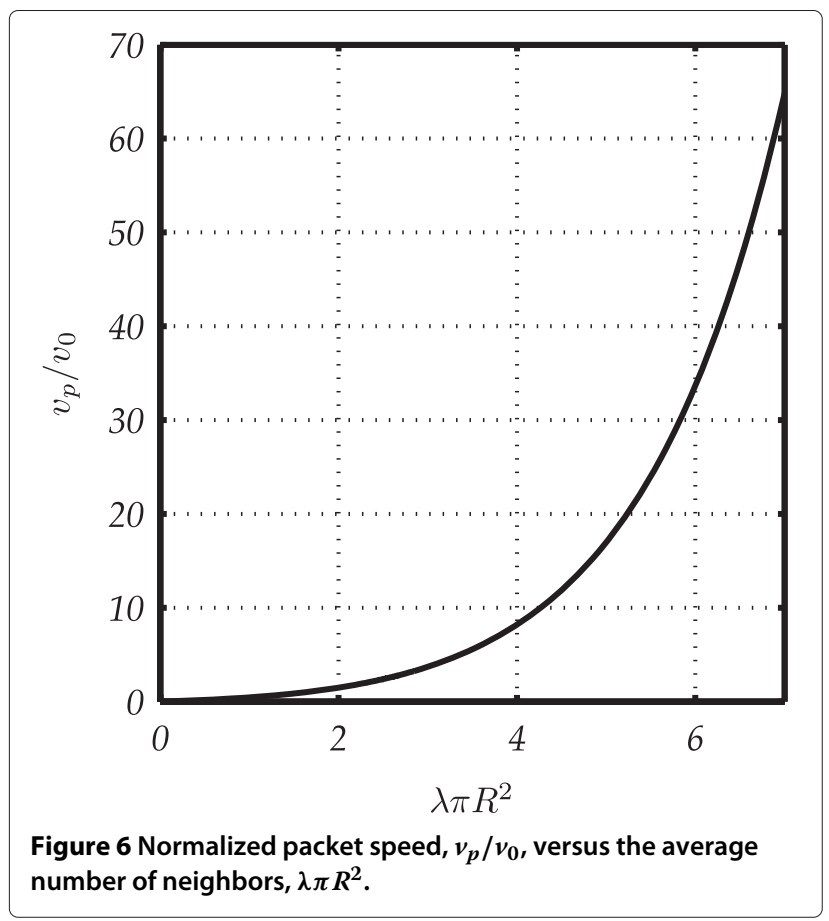

distance covered by the destination during the time it takes the packet to reach the destination. Clearly,

$$
\frac{R_{X}}{v_{0}}=\frac{D_{o d}+R_{X}}{v_{p}} \Rightarrow R_{X}=D_{o d} \frac{v_{0}}{v_{p}-v_{0}} .
$$

Let $D^{\text {DTFR }}$ be the delay in the delivery of the packet. It follows that

$$
D^{\mathrm{DTFR}}=\frac{R_{X}}{v_{0}}=\frac{D_{o d}}{v_{p}-v_{0}} \Rightarrow E\left(D^{\mathrm{DTFR}}\right)=\frac{E\left(D_{o d}\right)}{v_{p}-v_{0}} .
$$

Next, we calculate approximately the maximum throughput per node pair $T^{\text {DTFR }}$ that DTFR can support. To this end, we first calculate the average of the total cost $C^{\text {DTFR }}$ (in square meters) for the delivery of a packet to the destination. $C^{\text {DTFR }}$ is comprised of two terms: the cost $C_{A}$ up until the delivery of the packet to the FC, and the cost $C_{B}$ due to the transmissions taking place during the explosion and spread phases. The first term equals $c_{p} D_{o d}$. To calculate the second term, we first assume that the distance between the FC and the FEs is set to $R_{X}$, i.e., to the minimum that guarantees delivery of the packet given that nodes move with speed $v_{0}$ and the packet moves with speed $v_{p}$. Therefore, the cost of transmitting a single replica is $c_{p} R_{X}$. The cost of transmitting $L$ replicas consecutively is $L c_{p} R_{X}$. However, the replicas are not transmitted consecutively, but simultaneously. Therefore, many transmissions, particularly at locations close to the FC, can be combined, as they involve replicas of the same packet being transmitted from the same transmitter to the same receiver. Therefore, a more accurate approximation for the total cost during the spread and explosion phases is the total area that the replicas must cover during these phases, i.e., $\pi R_{X}^{2}$. Combining the two terms,

$$
\begin{aligned}
C^{\mathrm{DTFR}} & =C_{A}+C_{B}=c_{p} D_{o d}+\pi R_{X}^{2} \\
& =c_{p} D_{o d}+\pi D_{o d}^{2} \frac{v_{0}^{2}}{\left(v_{p}-v_{0}\right)^{2}} .
\end{aligned}
$$

Taking expectations,

$$
E\left(C^{\mathrm{DTFR}}\right)=c_{p} E\left(D_{o d}\right)+\pi E\left(D_{o d}^{2}\right) \frac{v_{0}^{2}}{\left(v_{p}-v_{0}\right)^{2}} .
$$

This average cost represents the average aggregate area of the footprints needed for the transmission of a single packet from the source to the destination.

To convert the average cost to the maximum throughput per node pair $T^{\text {DTFR }}$, we proceed as follows: as the node density is $\lambda$, each node is allocated on the average an area $\frac{1}{\lambda}$. As the area required for the transmission of a packet is $E\left(C^{\text {DTFR }}\right)$, it follows that each node can occupy the channel for a percentage of time equal to $\left(\frac{1}{\lambda}\right) / E\left(C^{\mathrm{DTFR}}\right)$, therefore

$$
\begin{aligned}
T^{\mathrm{DTFR}} & =\frac{r_{D}}{\lambda E\left(C^{\mathrm{DTFR}}\right)} \\
& =\frac{r_{D}}{\lambda}\left[c_{p} E\left(D_{o d}\right)+\pi E\left(D_{o d}^{2}\right) \frac{v_{0}^{2}}{\left(v_{p}-v_{0}\right)^{2}}\right]^{-1} .
\end{aligned}
$$

\subsection{Delay and throughput of BR}

BR operates similarly to DTFR, with the exception that the packet travels toward the destination, and never enters the explosion phase. Therefore, the trajectory that the packet follows is not a straight line. Finding its precise average length goes beyond the scope of this study. Noting that if $D_{o d} \gg R_{X}$ then this average length is approximately equal to $E\left(D_{o d}\right)$, we approximate it as $E\left(D_{o d}\right)$. It follows that

$$
\begin{aligned}
E\left(D^{\mathrm{BR}}\right) & =\frac{E\left(D_{o d}\right)}{v_{p}}, \quad E\left(C^{\mathrm{BR}}\right)=c_{p} E\left(D_{o d}\right), \\
T^{\mathrm{BR}} & =\frac{r_{D}}{\lambda c_{p} E\left(D_{o d}\right)} .
\end{aligned}
$$

\subsection{Discussion}

The effects that the basic environmental parameters have on the average delay and throughput of DTFR, as these were evaluated in the simulations, are consistent with the basic results of this section, i.e., (7) and (9). Indeed, (9) predicts that the average throughput will decrease when the size of the network, and hence $E\left(D_{o d}\right)$ and $E\left(D_{o d}^{2}\right)$, increase. Equation (7) also predicts that the 
average throughput will decrease when the size of the network increases, and so the average delay increases, but the TTL remains fixed. These predictions are consistent with Figure 3b. Equation (9) shows that increasing the transmission range $R$ increases the average throughput, because the second term in the brackets diminishes; this is verified by Figure 3c. Finally, (7) predicts that the delay decreases as the node speed increases, and this is consistent with Figure 3d which shows that, as the speed increases, the delivery rate increases, because more nodes arrive at their destinations before the TTL expires.

Furthermore, the analysis, in particular (6) and Figure 6, shows that there is a wide range of node degrees for which the network is not connected, and so traditional routing protocols cannot be used, but for which GLF ensures that the packet travels to the destination with a speed much larger than the node speed, and so can catch up with the destination quickly, while the cost of the Spread Phase remains manageable.

This last finding explains why the performance of DTFR is superior to that of SW. In more detail, for some values of the distance between the source and the destination, the maximum acceptable delay for the delivery of the packets, and the velocity of the nodes, the distance between the source and the destination cannot be covered by transport before the packet expires. SW spreads a number of copies in the area in which the nodes move, and one of the relays has to travel near the destination and transfer the packet there. A part of the distance between the source and the destination has to be covered by transport. A part of the distance between the source and the destination is covered by transmission, but if the packet travels distance, say $x$, by transmission, in one direction, due to symmetry the packet covers distance $x$ by transmission in all directions, and this has a large cost, at least $\pi x^{2}$, regardless if the distance $x$ is covered using many small or a few large hops. If the distance between the source and the destination is $s_{1}$, the delay in the above case is at least $\left(s_{1}-x\right) / v_{0}$. This bound on the delay-cost tradeoff of SW is very weak, but it gives an intuition as to why SW does not perform well for certain environment parameters. SW is designed to give a number of copies to relays in order to maximize the probability that a relay goes near the destination and delivers the packet, not to cover distance by transmission. In the above case, DTFR has delay $s_{1} /\left(v_{p}-v_{0}\right)$ and cost $c_{p} s_{1}+\pi s_{1}^{2} \frac{v_{0}^{2}}{\left(v_{p}-v_{0}\right)^{2}}$. Assume that the delay constraint is equal to the delay that DTFR can achieve. If we set $s_{1} /\left(v_{p}-v_{0}\right)=\left(s_{1}-x\right) / v_{0}$ it follows that $x=s_{1}\left(v_{p}-2 v_{0}\right) /\left(v_{p}-v_{0}\right)$. In this case, the cost of SW is at least $\pi s_{1}^{2}\left(v_{p}-2 v_{0}\right)^{2} /\left(v_{p}-v_{0}\right)^{2}$, while the cost of DTFR is less than this, for a number of values of the node degree. (For example, $\lambda \pi R^{2}=4.7$, which gives $v_{p}=13.75 v_{0}$ and $c_{p}=0.4294 R$.)

\section{Conclusions}

Our simulations reveal that DTFR performs better that SW in a variety of environment settings, that, notably, include VANETs. It also performs better than GeoCross and GeoDTN+Nav, two state-of-the-art routing protocols recently proposed specifically for use in VANETs. Our approximate analysis establishes how the packet delay and the per node throughput of DTFR scale with respect to various system parameters. We also show that in intermittently connected networks that are not very disconnected, DTFR can deliver packets faster than SW. We believe that both our simulation tool and the methods used in our analysis have wider applicability and may be of independent interest.

One case where the performance of DTFR is expected to suffer is when the road map has extensive local minima. Imagine, for example, the case of a city with the shape of a horseshoe (or, equivalently, the shape of a circular chain link missing a small sector). Any packet created at the one end of the horseshoe with its destination at the other end will be stuck at the Homing Phase, continuously using GLF; whenever, a node carries it far away from the end where it originated, a new node, lying towards the wrong end of the horseshoe, will soon appear to be closer to the destination, and the packet will be send toward the wrong end of the horseshoe. This problem has been identified early on in the context of geographic routing, and a number of solutions have been proposed. One solution is to use Trajectory-Based Forwarding (TBF) [33]. Using TBF, nodes employ geographical routing using, as a measure of the distance to the destination, not the Euclidean distance to the destination, but rather the progress along a trajectory. The trajectory stays within the bounds of the network, and thus local minima are avoided. Blažević et al. [34] propose Anchored Geodesic Packet Forwarding (AGPF); instead of the packet traveling directly to the destination using geographic routing, it travels to the destination by going through a sequence of anchors. Both TBF and AGPF can be integrated to DTFR: in the homing phase, instead of traveling along the straight line to the destination, a packet can travel along either a trajectory or a sequence of line segments created by anchors. Specifying the trajectory or the anchors can be achieved by an agent having access to a static map of the city, and the design is orthogonal to the various mechanisms of DTFR.

Although we have presented DTFR in the context of unicast traffic between nodes, we note that it can also support other types of traffic. Notably, it can be used for cooperative content sharing $[20,35]$. We now briefly elaborate on this $^{\mathrm{b}}$. In [20], a node $A$ disseminates information about data it has via k-hop broadcasting. A node $B$ that receives this information can send a query to $A$ about data it needs. Then $A$ can send the data to $B$. The query and the data are sent using AODV. This could be a possible application of 
DTFR. In the case of first time communication, no knowledge of the destination location is needed, as the message is send using limited flooding. After this, nodes have an estimate of the location of their destinations, and so can exchange the rest of the messages using DTFR.

\section{Appendix}

Let $C$ be a non-empty, convex, and compact subset of $\mathbb{R}^{2}$. We define the projection function $p_{C}: \mathbb{R} \rightarrow(0, \infty)$ as follows: if $\chi \in \mathbb{R}$, then $p_{C}(\chi)>0$ is the minimum width that an infinite closed strip, inclined with respect to the $x$ axis by an angle $\chi$, can have and at the same time have $C$ as its subset, as depicted in Figure 7. Observe that $p_{C}(\chi)=$ $p_{C}(\chi+\pi)$ for all $\chi \in \mathbb{R}$. For the semicircular forwarding region $F$ of our analysis, straightforward geometry shows that

$$
p_{F}(\chi)=R(1+|\cos \chi|) .
$$

Lemma 1. Let $C$ be a non-empty, convex, and compact subset of $\mathbb{R}^{2}$ with projection function $p_{C}(\chi)$. Let $\mathbb{R}^{2}$ be uniformly covered by nodes distributed, at time 0 , according to a spatial Poisson process of density $\lambda$, all moving with a common speed $\mathbf{v}$ of magnitude $v_{0}$ and direction with respect to the $x$ axis equal to $\chi$. Then, nodes enter $C$ through its boundary according to a Poisson process with rate equal to $\gamma(\chi)=\nu_{0} p_{C}(\chi) \lambda$.

Proof. Consider Figure 8 and focus on a time instant $t_{0}$ and a time interval $\left[t_{0}, t_{0}+T\right]$. The nodes that enter the lightly shaded set $C$ during this interval are exactly those

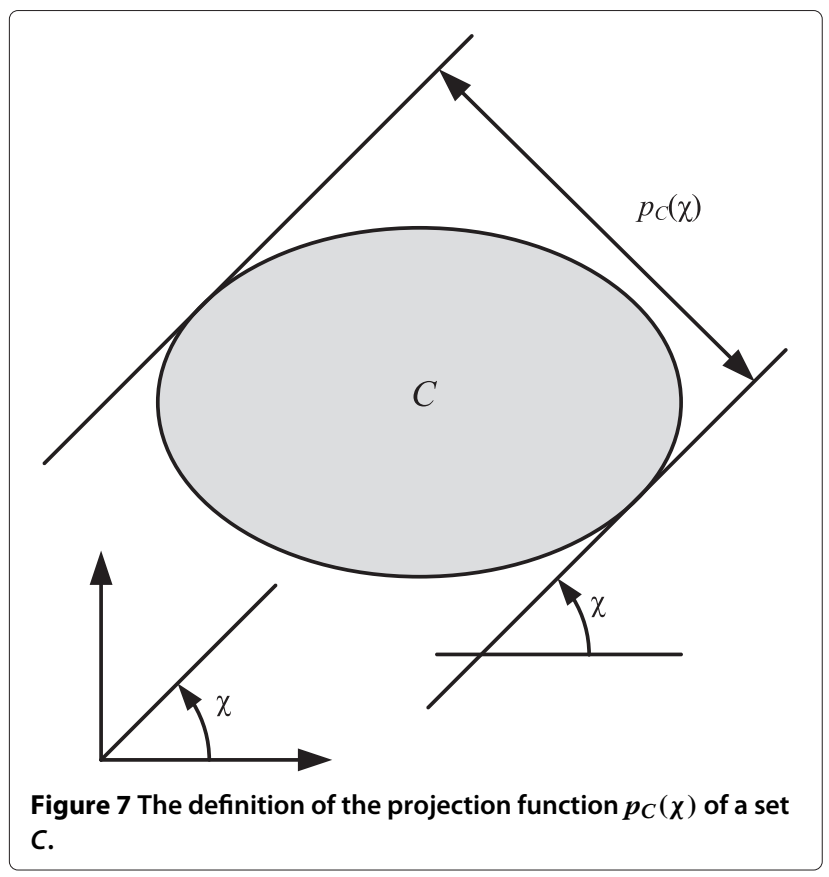

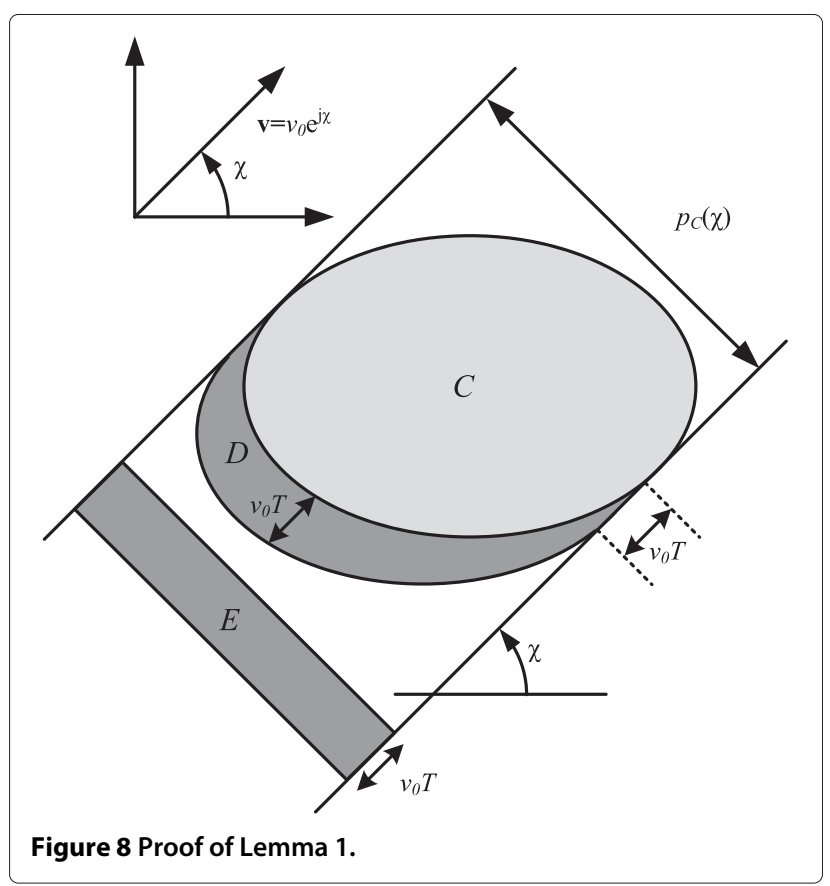

that at time $t_{0}$ are on the darker shaded set $D$, whose nonlinear boundaries are parallel to each other and are on a distance of $v_{0} T$ from each other. Since the nodes follow a spatial Poisson distribution at time $t=0$, they will also follow a spatial Poisson distribution, with the same density, at time $t_{0}$. Therefore, the nodes within $D$ are Poisson distributed, with mean equal to $\lambda$ multiplied by the area of $D$. Note, however, that this area equals the area $p_{C}(\chi) \nu_{0} T$ covered by the rectangle $E$. Therefore, the average number of nodes entering $C$ in the time interval $\left[t_{0}, t_{0}+T\right]$ is $v_{0} p_{C}(\chi) \lambda T$. Finally, also note that the numbers of nodes arriving at non-overlapping time intervals are independent, because they are due to the existence of nodes, at time $t=0$, at non-overlapping subsets of $\mathbb{R}^{2}$. It follows that the arrival process of nodes in $C$ satisfies the definition of a Poisson process [36] with rate $\gamma(\chi)=v_{0} p_{C}(\chi) \lambda$.

Lemma 2. Let $L$ be a linear segment of length $l$, parallel to the $y$-axis. Let $L$ move with a velocity $\mathbf{v}_{L}$ of magnitude $v_{0}$, forming an angle $\theta \in[0, \pi]$ with the positive $x$-axis. Let $\mathbb{R}^{2}$ be covered with nodes placed, at time $t=0$, according to a spatial Poisson process of density $\lambda$, and moving with velocity vectors of magnitude $v_{0}$ and directions uniformly distributed in $[0,2 \pi]$, independently of each other. Then, the process with which nodes cross $L$ through its side $L_{1}$ looking at the positive $x$-axis is Poisson, with rate

$$
\gamma_{L}(\theta)=\frac{\lambda l \nu_{0}}{\pi}[\sin \theta+(\pi-\theta) \cos \theta]
$$


Proof. Assume, for now, that all nodes have the same velocity vector $\mathbf{v}_{N}$, and travel toward the same angle $\phi \in$ $[0,2 \pi]$. Using phasor notation, $\mathbf{v}_{N}=v_{0} e^{j \phi}$ and $\mathbf{v}_{L}=v_{0} e^{j \theta}$. The setting appears in Figure 9. We will specify the process with which nodes cross $L$ (entering from side $L_{1}$ ) in this case.

Observe, first, that we must have $\phi \in[\theta, 2 \pi-\theta]$, otherwise the nodes arrive at $L$ from the other side, $L_{2}$. Also observe that the relative velocity of the nodes with respect to $L$ is

$$
\begin{aligned}
\mathbf{v}_{N}-\mathbf{v}_{L} & =v_{0} e^{j \phi}-v_{0} e^{j \theta}=v_{0} e^{j \phi}+v_{0} e^{j(\theta+\pi)} \\
& =2 v_{0} \cos \left(\frac{\pi-\phi+\theta}{2}\right) e^{j\left(\frac{\phi+\theta+\pi}{2}\right)} \\
& =2 v_{0} \sin \left(\frac{\phi-\theta}{2}\right) e^{j\left(\frac{\phi+\theta+\pi}{2}\right)} .
\end{aligned}
$$

Let us move to the coordinate system where $L$ remains stationary and parallel to the $y$-axis. Consider a time interval $\left[t_{0}, t_{0}+T\right]$. The nodes crossing $L$ from $L_{1}$ are exactly those that at $t_{0}$ are in the shaded rectangle of Figure 9. The number of those is a Poisson random variable with average $\lambda$ multiplied by the area of the rectangle $\left(2 v_{0} T \sin \left(\frac{\phi-\theta}{2}\right)\right) \times l \times \sin \left(\frac{\phi+\theta}{2}\right)$. To conclude, the nodes arriving in the interval $\left[t_{0}, t_{0}+T\right]$ are Poisson distributed with average $2 v_{0} \lambda l \sin \left(\frac{\phi-\theta}{2}\right) \sin \left(\frac{\phi+\theta}{2}\right) T$. Furthermore, the arrivals at non-overlapping time intervals are independent, because they are caused by the existence of nodes, during time $t_{0}$, at non-overlapping subsets of $\mathbb{R}^{2}$. It follows [36] that the arrival process is Poisson with rate $2 v_{0} \lambda l \sin \left(\frac{\phi-\theta}{2}\right) \sin \left(\frac{\phi+\theta}{2}\right)$.

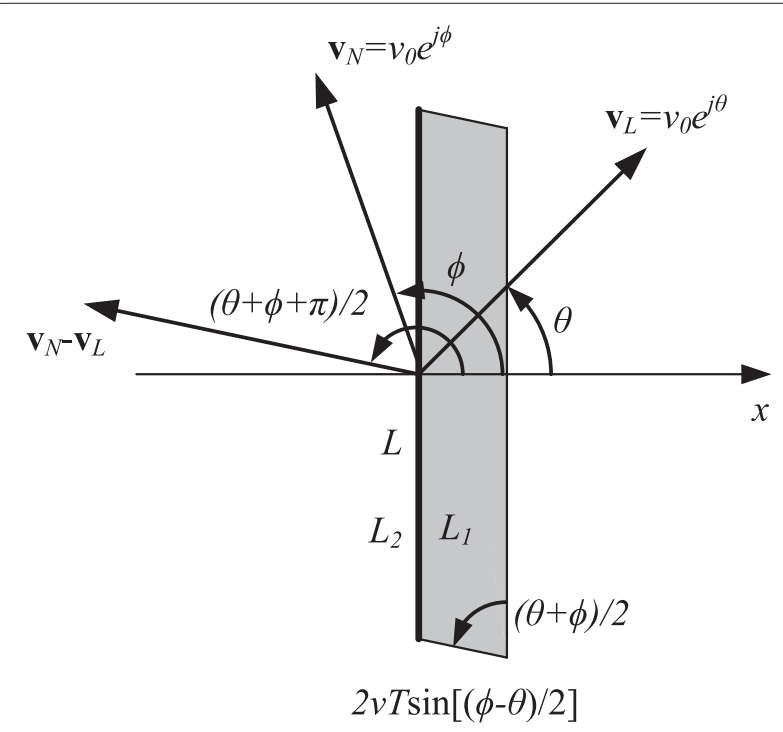

Figure 9 The setting of Lemma 2.
However, nodes do not have a fixed direction $\phi$, but rather the direction of each is uniformly distributed and independent of the directions of the rest. It follows from the previous case that the arrivals of nodes with direction in the incremental range $[\phi, \phi+d \phi]$ form a Poisson process with incremental rate $2 v_{0} \frac{\lambda d \phi}{2 \pi} l \sin \left(\frac{\phi-\theta}{2}\right) \sin \left(\frac{\phi+\theta}{2}\right)$. As the summation of multiple Poisson processes is a Poisson process with rate equal to the sum of the rates, it follows that the arrival process from all angles is a Poisson process with rate given by the integral

$$
\gamma_{L}(\theta)=\int_{\theta}^{2 \pi-\theta} \frac{\lambda l \nu_{0}}{\pi} \sin \left(\frac{\phi-\theta}{2}\right) \sin \left(\frac{\phi+\theta}{2}\right) d \phi .
$$

Calculating the integral, we arrive at (11).

We are now ready to proceed with the proof of Equations (5), which is organized in three parts.

Part 1: Assume, for now, that $F$ is moving with a constant velocity $\mathbf{v}_{F}$ of magnitude $v_{0}$ and direction (with respect to the $x$-axis) equal to $\phi$, where $\phi \in[-\pi, \pi]$. Let also $\mathbb{R}^{2}$ be filled with nodes, all with a common velocity $\mathbf{v}_{N}$ of magnitude $v_{0}$ and direction, with respect to the $x$-axis, equal to $\theta$, where $\theta \in[-\pi, \pi]$. At time $t=0$, nodes are placed on $\mathbb{R}^{2}$ according to a spatial Poisson process with density $\lambda$. The setting appears in Figure 10. In this setting, the arrival process of nodes in $F$ through its boundary is a Poisson process with rate equal to

$$
\gamma(\theta, \phi)=2 v_{0} R \lambda\left|\sin \left(\frac{\theta-\phi}{2}\right)\right|\left(1+\left|\sin \left(\frac{\phi+\theta}{2}\right)\right|\right) .
$$

Indeed, in phasor notation, $\mathbf{v}_{N}=v_{0} e^{j \theta}$ and $\mathbf{v}_{F}=v_{0} e^{j \phi}$, and the relative velocity of the nodes as perceived by $F$ is

$$
\mathbf{v}_{N}-\mathbf{v}_{F}=v_{0} e^{j \theta}+v_{0} e^{j(\phi+\pi)}=2 v_{0} \sin \left(\frac{\theta-\phi}{2}\right) e^{j \frac{\phi+\theta+\pi}{2}} .
$$

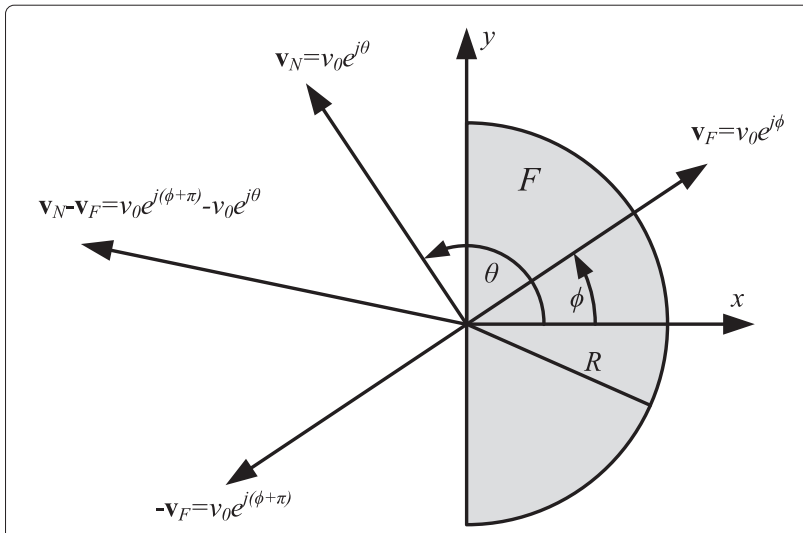

Figure 10 The semicircle used in the proof of Equation (5). 
Therefore, the magnitude of the relative velocity is $2 v_{0}\left|\sin \left(\frac{\theta-\phi}{2}\right)\right|$, and the angle of incidence of the nodes on $F$ is either $\frac{\phi+\theta+\pi}{2}$ or $\frac{\phi+\theta+\pi}{2}-\pi=\frac{\phi+\theta-\pi}{2}$, depending on the sign of $\sin \left(\frac{\theta-\phi}{2}\right)$. As the two possibilities for the angle of incidence differ by $\pi$, they give the same value of the projection function. It follows from Lemma 1 that

$$
\gamma(\theta, \phi)=2 \lambda v_{0}\left|\sin \left(\frac{\theta-\phi}{2}\right)\right| p_{F}\left(\frac{\phi+\theta+\pi}{2}\right),
$$

and substituting $p_{F}(\cdot)$ from (10) the result (12) follows.

Part 2: We now modify the setting of Part 1 to assume that each node is moving toward a direction $\Theta$ that is uniformly chosen in $[-\pi, \pi]$, independently of the directions of all other nodes. In this setting, the arrival process is again a Poisson process with rate equal to

$$
\gamma(\phi)=\frac{\nu_{0} R \lambda}{\pi}[4+(\pi-2|\phi|) \cos \phi+2 \sin |\phi|] .
$$

This is due to the facts that, first, each incremental range of node velocity angles, $[\theta, \theta+d \theta]$ creates a Poisson arrival process and, second, the process consisting of the arrivals of any number of Poisson processes is still a Poisson process, with a rate equal to the sum of the incremental rates, i.e., the integral

$$
\begin{aligned}
\gamma(\phi) & =\frac{1}{2 \pi} \int_{-\pi}^{\pi} \gamma(\theta, \phi) d \theta \\
& =\frac{\nu_{0} R \lambda}{\pi} \int_{-\pi}^{\pi}\left|\sin \left(\frac{\theta-\phi}{2}\right)\right|\left(1+\left|\sin \left(\frac{\phi+\theta}{2}\right)\right|\right) d \theta
\end{aligned}
$$

After straightforward calculations, (13) follows.

Part 3: Let us now move to the setting of Equations (5): Let $F$ be empty of nodes and at time $t=0$ centered at the origin. Let the directions of both $F$ and all nodes be randomly and uniformly distributed in $[-\pi, \pi]$, and let $\Phi$ be the random direction of $F$. In this setting, the arrival process of nodes at the boundary of $F$ is a conditional Poisson process [36]. Indeed, if we condition on $\Phi$, we fall into Part 2, in which the node arrival process is Poisson, with rate given by (13). Observe that conditioning on $M$ does not affect the statistics of new arrivals, as these are coming from regions that do not overlap with the interior of $F$ at $t=0$.

To calculate $E\left(D_{1} \mid M\right), E\left(X_{1} \mid M\right)$, and $E\left(C_{1} \mid M\right)$, we condition on $\Phi$. We start with $E\left(D_{1} \mid M\right)$, noting that

$$
\begin{aligned}
E\left(D_{1} \mid M\right) & =E\left(E\left(D_{1} \mid \Phi, M\right)\right) \\
& =\frac{1}{2 \pi} \int_{-\pi}^{\pi} E\left(D_{1} \mid \Phi=\phi, M\right) d \phi \\
& =\frac{1}{\pi} \int_{0}^{\pi} E\left(D_{1} \mid \Phi=\phi, M\right) d \phi,
\end{aligned}
$$

where in the last equation we used the fact that, due to symmetry, the function $E\left(D_{1} \mid \Phi=\phi, M\right)$ is even. As discussed, conditioned on $\Phi=\phi$, the node arrival process is a Poisson process with rate $\gamma(\phi)$ given in (13), and hence $E\left(D_{1} \mid \Phi=\phi, M\right)=\frac{1}{\gamma(\phi)}$. Combining everything, it follows that

$$
\begin{aligned}
& E\left(D_{1} \mid M\right)=\frac{I_{1}}{v_{0} R \lambda}, \\
& I_{1} \triangleq \int_{0}^{\pi} \frac{d \phi}{4+(\pi-2 \phi) \cos \phi+2 \sin \phi} \simeq 0.4817 .
\end{aligned}
$$

Next, we calculate $E\left(X_{1} \mid M\right)$, noting that $E\left(X_{1} \mid M\right)=$ $E\left(X_{T} \mid M\right)+E\left(X_{A} \mid M\right)$. We first note that

$$
\begin{aligned}
E\left(X_{A} \mid M\right) & =E\left(E\left(X_{A} \mid \Phi, M\right)\right) \\
& =\frac{1}{2 \pi} \int_{-\pi}^{\pi} E\left(X_{A} \mid \Phi=\phi, M\right) d \phi \\
& =\frac{1}{\pi} \int_{0}^{\pi} E\left(X_{A} \mid \Phi=\phi, M\right) d \phi,
\end{aligned}
$$

where the last equality is due to symmetry. Now observe that $\gamma(\phi)=\gamma(\pi-\phi)$ for all $\phi \in[0, \pi]$. It follows that $E\left(X_{A} \mid \Phi=\phi, M\right)=-E\left(X_{A} \mid \Phi=\pi-\phi, M\right)$, and therefore $E\left(X_{A} \mid M\right)$ is zero. It follows that $E\left(X_{1} \mid M\right)=E\left(X_{T} \mid M\right)$.

Furthermore,

$$
\begin{aligned}
E\left(X_{T} \mid M\right) & =E\left(E\left(X_{T} \mid \Phi, M\right)\right) \\
& =\frac{1}{2 \pi} \int_{-\pi}^{\pi} E\left(X_{T} \mid \Phi=\phi, M\right) d \phi \\
& =\frac{1}{\pi} \int_{0}^{\pi} E\left(X_{T} \mid \Phi=\phi, M\right) d \phi,
\end{aligned}
$$

where in the last equation we used symmetry. As discussed, conditioned on $\Phi=\phi$, the node arrival process is a Poisson process with rate $\gamma(\phi)$ given in (13). This Poisson process can be broken down to a set of independent, incremental Poisson processes, each one corresponding to the node arrivals in the semicircle through an incremental arc $[\chi, \chi+d \chi]$ along its circumference, where $\chi \in[-\pi / 2, \pi / 2]$, and a last one, independent of the rest, corresponding to the arrivals through the linear part. It follows that the probability that there is an arrival through such an arc $\chi \in[-\pi / 2, \pi / 2]$ is equal to the incremental rate of arrivals there, $d \gamma(\chi)$ over the aggregate arrival rate $\gamma(\phi)$. Therefore, and noting that arrivals through the linear part do not contribute to $E\left(X_{T} \mid \Phi=\phi, M\right)$, we have

$$
E\left(X_{T} \mid \Phi=\phi, M\right)=\int_{-\pi / 2}^{\pi / 2} R \cos \chi \frac{d \gamma(\chi)}{\gamma(\phi)} .
$$

Observe, however, that $d \gamma(\chi)$ is equal to the arrival rate through a linear segment of length $R d \chi$ and moving toward an angle $|\chi-\phi|$, with respect to its vertical. 
Therefore, Lemma 2 applies. Taking into account that $|\chi-\phi|$ might be greater than $\pi$, it follows that

$$
\begin{aligned}
& d \gamma(\chi)=\frac{R \lambda v_{0}}{\pi} h(|\chi-\phi|) d \chi, \quad \text { where } \\
& h(x) \triangleq \begin{cases}\sin x+(\pi-x) \cos x, & 0 \leq x \leq \pi, \\
h(2 \pi-x), & \pi<x \leq 2 \pi .\end{cases}
\end{aligned}
$$

Putting everything together, it follows that

$$
\begin{aligned}
& E\left(X_{1} \mid M\right)=I_{2} R \\
& I_{2} \triangleq \int_{0}^{\pi} \frac{\frac{1}{\pi} \int_{-\pi / 2}^{\pi / 2} h(|\chi-\phi|) \cos \chi d \chi}{4+(\pi-2 \phi) \cos \phi+2 \sin \phi} d \phi \simeq 0.3894
\end{aligned}
$$

To calculate $E\left(C_{1} \mid M\right)=\pi E\left(X_{T}^{2}+Y_{T}^{2} \mid M\right)$, note that

$$
\begin{aligned}
E\left(X_{T}^{2}+Y_{T}^{2} \mid M\right) & =E\left(E\left(X_{T}^{2}+Y_{T}^{2} \mid \Phi, M\right)\right) \\
& =\frac{1}{\pi} \int_{0}^{\pi} E\left(X_{T}^{2}+Y_{T}^{2} \mid \Phi=\phi, M\right) d \phi,
\end{aligned}
$$

The value of $X_{T}^{2}+Y_{T}^{2}$ depends on whether the arriving node comes through the linear segment of the semicircle or not. The probability $P(S)$ of the event $S$ that the arrival will be through the linear segment equals the rate of arrivals through the linear segment, as specified by Lemma 2, over the total rate of arrivals $\gamma(\phi)$, as specified by (13). Therefore,

$$
\begin{aligned}
P(S) & =\frac{\frac{2 \lambda R v_{0}}{\pi}[\sin (\pi-\phi)+\phi \cos (\pi-\phi)]}{\frac{\lambda R v_{0}}{\pi}[4+(\pi-2 \phi) \cos \phi+2 \sin \phi]} \\
& =\frac{2(\sin \phi-\phi \cos \phi)}{4+(\pi-2 \phi) \cos \phi+2 \sin \phi} .
\end{aligned}
$$

Having $P(S)$, we note that

$$
\begin{aligned}
E\left(X_{T}^{2}+Y_{T}^{2} \mid \Phi=\phi, M\right) & =[1-P(S)] R^{2}+P(S) \int_{-R}^{R} \frac{y^{2} d y}{2 R} \\
& =R^{2}\left(1-\frac{2}{3} P(S)\right) .
\end{aligned}
$$

Combining everything, it follows that

$$
\begin{aligned}
& E\left(C_{1} \mid M\right)=I_{3} R^{2} \\
& I_{3} \triangleq \int_{0}^{\pi}\left(1-\frac{2}{3} \times \frac{2(\sin \phi-\phi \cos \phi)}{4+(\pi-2 \phi) \cos \phi+2 \sin \phi}\right) d \phi \\
& \simeq 2.3317 .
\end{aligned}
$$

\section{Endnotes}

${ }^{\text {a }}$ The interested reader is referred to $[37,38]$, where the packet speed in a DTN is investigated without the use of this assumption (although various others are made). There, however, the focus is exclusively on the topic of the packet speed in DTNs.

${ }^{\mathrm{b}} \mathrm{A}$ detailed exposition is subject for future work.

\section{Competing interests}

The authors declare that they have no competing interests.

\section{Acknowledgements}

This research has been co-financed by the European Union (European Social Fund - ESF) and Greek national funds through the Operational Program "Education and Lifelong Learning" of the National Strategic Reference Framework (NSRF) Research Funding Program THALES: Investing in knowledge society through the European Social Fund. We would like to thank Pei-Chun Cheng for many useful discussions about the implementation and operation of GeoDTN+Nav, Savvas Gitzenis for suggestions on the presentation of the material, and the anonymous reviewers for their many insightful comments.

\section{Author details}

${ }^{1}$ Department of Electrical and Computer Engineering, University of Cyprus, 75 Kallipoleos Avenue, 1678 Nicosia, Cyprus. ${ }^{2}$ Department of Informatics, Athens University of Economics and Business, 76 Patision Avenue, 10434 Athens, Greece.

Received: 23 October 2011 Accepted: 7 January 2013 Published: 7 February 2013

\section{References}

1. TSmall, ZJ Haas, in Proceedings of the ACM MobiHOC. The shared wireless infostation model - a new ad hoc networking paradigm (or where there is a whale, there is a way), Annapolis, MD, 2003), pp. 233-244

2. RC Shah, S Roy, S Jain, W Brunette, Data MULEs: modeling and analysis of a three-tier architecture for sparse sensor networks. Ad Hoc Netw. 1(6), 215-233 (2003)

3. N Laoutaris, G Smaragdakis, P Rodriguez, R Sundaram, in Proceedings of the ACM Sigmetrics 2009. Delay tolerant bulk data transfers on the internet, Seattle, WA, 2009), pp. 229-238

4. M Grossglauser, D Tse, in Proceedings of the IEEE INFOCOM, vol. 3. Mobility increases the capacity of ad-hoc wireless networks, Anchorage, AL, 2001), pp. 1360-1369

5. SToumpis, AJ Goldsmith, in Proceedings of the IEEE INFOCOM, vol. 1. Large wireless networks under fading, mobility, and delay constraints, Hong Kong, China, 2004)

6. T Spyropoulos, K Psounis, CS Raghavendra, Efficient routing in intermittently connected mobile networks: the single-copy case. IEEE Trans. Netw. 16, 63-76 (2008)

7. TSpyropoulos, K Psounis, CS Raghavendra, Efficient routing in intermittently connected mobile networks: the multiple-copy case. IEEE Trans. Netw. 16, 77-90 (2008)

8. J LeBrun, CN Chiah, D Ghosal, M Zhang, in Proceedings of the IEEE VTC Spring, vol. 4. Knowledge-based opportunistic forwarding in vehicular wireless ad hoc networks, Florence, Italy, 2005), pp. 2289-2293

9. C Lochert, M Mauve, H Fubler, H Hartenstein, Geographic routing in city scenarios. ACM SIGMOBILE Mob. Comput. Commun. Rev. 9, 69-72 (2005)

10. KC Lee, PC Cheng, M Gerla, GeoCross: a geographic routing protocol in the presence of loops in urban scenarios. Ad Hoc Netw. 8, 474-488 (2010)

11. PC Cheng, KC Lee, M Gerla, J Harri, GeoDTN+Nav: geographic DTN routing with navigator prediction for urban vehicular environments. Mob. Netw. Appl. 15, 61-82 (2010)

12. Wikipedia. [http://en.wikipedia.org/wiki/Fireworks\#Palm]

13. H Wu, R Fujimoto, R Guensler, M Hunter, Pennsylvania Philadelphia USA, in ACM MOBICOM. MDDV: a mobility-centric data dissemination algorithm for vehicular networks, (2004), pp. 47-56

14. B Karp, HT Kung, in MobiCom. GPSR: greedy perimeter stateless routing for wireless networks, Boston, MA, 2000), pp. 243-254

15. E Kuiper, $\mathbf{S}$ Nadjm-Tehrani, Geographical routing with location service in intermittently connected MANETs. IEEE Trans. Veh. Technol. 60(2), 592-604 (2011)

16. $\mathrm{CH} \mathrm{Ng}, \mathrm{KC} \mathrm{Sia}, \mathrm{CH}$ Chan, in 12th International World Wide Web Conference Peer clustering and firework query model in the peer-to-peer network, (2003)

17. KC Sia, CH Ng, CH Chan, I King, in 2nd International Workshop on Peer-to-Peer Systems. P2P content-based query routing using firework query model, (2003) 
18. L Law, S Krishnamurthy, M Faloutsos, in IFIP Networking 2005. Fireworks: an adaptive group communications protocol for mobile ad hoc networks, Waterloo, Canada, 2005), pp. 853-868

19. A Sidera, S Toumpis, in Proceedings of the IFIP MedHocNet 2011. DTFR: a geographic routing protocol for wireless delay tolerant networks, Favignana Island, Sicily, Italy, 2011), pp. 33-40

20. K Lee, SH Lee, R Cheung, U Lee, M Gerla, in Mobile Networking for Vehicular Environments. First experience with CarTorrent in a real vehicular ad hoc network testbed, Anchorage, AK, USA, 2007), pp. 109-114

21. M Heissenbuettel, T Braun, M Waelchli, T Bernoulli, Evaluating the limitations of and alternatives in beaconing. Ad Hoc Netw. 5, 558-578 (2007)

22. S Heimlicher, M Karaliopoulos, H Levy, T Spyropoulos, in IEEE INFOCOM. On leveraging partial paths in partially-connected networks, Rio de Janeiro, Brazil, 2009), pp. 55-63

23. A Sidera, S Toumpis, Delay tolerant firework routing. Technical report, [pages.cs.aueb.gr/ toumpis/dtns/technical_report.pdf]

24. Very Large DTN Simulator (VL-DTN-S). [http://pages.cs.aueb.gr/ toumpis/ dtns.html]

25. ns-3 homepage. [http://www.nsnam.org]

26. OMNET++ community homepage. [http://www.omnetpp.org]

27. A Karänen, J Ott, T Kärkkäinen, in Proceedings of the Simutools 2009. The ONE simulator for DTN protocol evaluation, Rome, Italy, 2009)

28. OR Helgason, KV Jónsson, in Proceedings of the OMNeT++. Opportunistic networking in OMNetT++, Marseille, France, 2008)

29. T Rappaport, Wireless Communications: Principles and Practice. (Prentice Hall, Upper Saddle River, 2002)

30. P Sommer, R Wattenhofer, in Proceedings of the ACM ISPN. Gradient clock synchronization in wireless sensor networks, San Francisco, CA, 2009), pp. $37-48$

31. P Gupta, PR Kumar, The capacity of wireless networks. IEEE Trans. Inf. Theory. 46(2), 388-404 (2000)

32. R Nelson, L Kleinrock, The spatial capacity of a slotted ALOHA, multihop packet radio network with capture. IEEE Trans. Commun. COM-32(6), 684-694 (1984)

33. D Niculescu, B Nath, in Proceedings of the ACM MobiCom. Trajectory based forwarding and its applications, (San Diego, CA, 2003), pp. 260-272

34. L Blažević, S Giordano, J-Y Le Boudec, Self organized terminode routing. Cluster Comput. 5, 205-218 (2002)

35. V Lenders, G Karlsson, M May, in IEEE SECON. Wireless ad hoc podcasting, San Diego, CA, 2007), pp. 273-283

36. SM Ross, Introduction to Probability Models, 8th edn. (Academic Press, Boston, 2003)

37. P Jacquet, B Mans, G Rodolakis, in Proceedings of the IEEE INFOCOM. Information propagation speed in mobile and delay tolerant networks, (Rio de Janeiro, Brazil, 2009), pp. 5001-5015

38. E Baccelli, P Jacquet, B Mans, G Rodolakis, in Proceedings of the IEEE INFOCOM. Information propagation speed in bidirectional vehicular delay tolerant networks, Shanghai, China, 2011)

\section{Submit your manuscript to a SpringerOpen ${ }^{\mathcal{O}}$ journal and benefit from:}

- Convenient online submission

- Rigorous peer review

- Immediate publication on acceptance

- Open access: articles freely available online

- High visibility within the field

- Retaining the copyright to your article

Submit your next manuscript at $\boldsymbol{~ s p r i n g e r o p e n . c o m ~}$ 